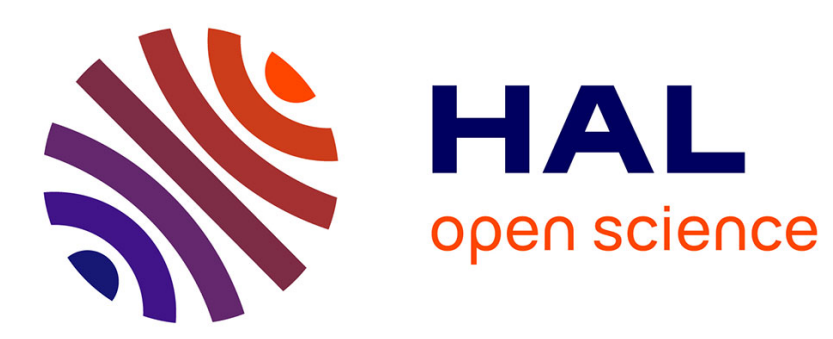

\title{
A Reduced Differential Model of the Electrical Activity of Cardiac Purkinje Fibres
}

\author{
Karima Djabella, Michel Sorine
}

\section{To cite this version:}

Karima Djabella, Michel Sorine. A Reduced Differential Model of the Electrical Activity of Cardiac Purkinje Fibres. 28 th Annual International Conference of the IEEE Engineering in Medicine and Biology Society - EMBS'06, Aug 2006, New York, pp.4167-4170, 10.1109/IEMBS.2006.259468 . inria00001197v2

\section{HAL Id: inria-00001197 https://hal.inria.fr/inria-00001197v2}

Submitted on 4 Apr 2006

HAL is a multi-disciplinary open access archive for the deposit and dissemination of scientific research documents, whether they are published or not. The documents may come from teaching and research institutions in France or abroad, or from public or private research centers.
L'archive ouverte pluridisciplinaire HAL, est destinée au dépôt et à la diffusion de documents scientifiques de niveau recherche, publiés ou non, émanant des établissements d'enseignement et de recherche français ou étrangers, des laboratoires publics ou privés. 


\title{
A Reduced Differential Model of the Electrical Activity of Cardiac Purkinje Fibres
}

\author{
Karima Djabella and Michel Sorine
}

\begin{abstract}
A reduced order differential model of cardiac Purkinje fibres action potential, with only eight state variables, is presented. Its structure, derived from basic physical principles can be used for the main other cardiac cell types, a useful property for some model-based signal or image processing applications. The electrical activity of cardiac Purkinje fibres is reconstructed using particular values of the parameters. This model of the membrane excitation mechanism and intracellular calcium dynamics describes the principal ionic current underlying autorhythmicity; calcium uptake and release from the sarcoplasmic reticulum; effects of the binding of calcium on myoplasmic proteins which affect the Nernst potential of calcium, and then the membrane potential. The model allows realistic modelling of cardiac Purkinje fibres action potential, total ionic current, CICR dependence on intracellular calcium concentrations. Simulations illustrate the role of the inward sodium current as the dominant mechanism underlying pacemaker depolarization during spontaneous activity.
\end{abstract}

\section{INTRODUCTION}

The cardiac Purkinje fibres constitute the conduction system in the ventricles and, being capable of self-excitation, they act as an accessory pacemaker. They spontaneously and rhythmically generate action potentials (AP) resulting in muscle contraction. Accurate modelling of their AP and pacemaker activity is difficult due to the complexity of the excitation mechanisms involving a large number of different ionic currents. The first models of AP in cardiac Purkinje fibres (Noble in 1962 [1], updated by McAllister et al [2]) used the Hodgkin \& Huxley's formulation of the properties of excitable membranes to describe the long lasting AP and pacemaker activity. DiFrancesco and Noble developed a new model of Purkinje fibres electrical activity [3] that was the first cardiac cell model incorporating ionic exchangers, pumps and concentration changes. These models have their respective advantages and have proven to be useful in investigating a variety of heart diseases. They differ in many details from models of atrial or ventricular cardiac cell.

As for other biochemical systems, an apparent paradox in cardiac cell modelling is that the knowledge on cell structure imbedded in models is rather rich but it is quite impossible to experimentally validate the forms of the different nonlinearities used in detailed models and their parameters are poorly known. In fact, mass and electrical charge conservation laws are direct applications of fundamental physical principles, but many model details are Ad-hoc descriptions of observed behaviors, differing with the authors even for the same cell. Nevertheless, apparently different models, after some

\footnotetext{
The authors are with INRIA-Rocquencourt, B.P. 105 , 78153 Le Chesnay Cedex, France. Karima.Djabella, Michel.Sorinedinria.fr
}

parameter tuning, agree very well with experimental data. Identifying model structures leading to both these parameter sensitivity and robustness of model properties is then of particular interest. This may allow to retain only the necessary details in a model intended for a given task. This is what is attempted here to facilitate the development of modelbased signal or image processing applications where $0 \mathrm{D}$ or 3D models of the heart and associated inverse problems are considered [4]. No general technique is available to determine such structure. Only in very simple situations generic models are known such as the ubiquitous FitzHugh Nagumo equations for two-state variable models. Our approach is then to reduce the state space dimension of different cardiac cell models trying to reorganize them with a common structure.

In this paper, a reduced differential model for cardiac Purkinje fibres AP is proposed. It shares the same structure than the reduced model of ventricular cell in [5]. It incorporates some myoplasmic calcium proteins which are involved in the process of excitation-contraction coupling in ventricular cells and are useful here, as they affect the Nernst potential of calcium, and then the membrane potential. It has only eight state variables. Three variables are used to describe the membrane (membrane potential and two gate variables of ionic channels), taking into consideration the dynamics of the main ionic currents (inward sodium, Ltype calcium and outward potassium), $\mathrm{Na}^{+} / \mathrm{Ca}^{2+}$ exchangers and $\mathrm{Na}^{+} / \mathrm{K}^{+}$pumps, derived using physical principles and conservation laws [6]. The remaining five variables are associated with the fluid compartment model accounting for intracellular changes in concentrations of $\mathrm{Na}^{+}, \mathrm{K}^{+}$[7] and the main processes that regulate intracellular calcium concentrations: release and uptake by the sarcoplasmic reticulum (SR), buffering in the SR [8] and in the bulk cytosol [9].

The inherent ability of Purkinje fibres to generate a depolarizing unstable resting potential leading to automaticity can be caused either by decreasing outward current (the timedependant decrease in delayed outward potassium current $I_{K}$ ) or the development of a net inward ion flux immediately following AP repolarization [10]. The precise contribution of underlying membrane currents to the net inward flow still remains the subject of controversy (three main hypotheses of pacemaker generation have emerged, the inward hyperpolarization activated current $\left(i_{f}\right)$ which is normally carried by $\mathrm{Na}^{+}$and $\mathrm{K}^{+}$, the sustained inward current $i_{s t}$, where the major charge carrier is believed to be $\mathrm{Na}^{+}$and a $\mathrm{Ca}^{2+}$ "window" current). Despite its moderate number of state variables, the model includes the main processes thought to be important in pacemaker activity and it incor- 
porates the most important currents underlying pacemaker depolarization in Purkinje cells. After model tuning, the mechanisms underlying pacemaker depolarization have been investigated, and the inward sodium current appears to be the most dominant one. The model successfully reproduces experimentally observed cardiac Purkinje fibres pacemaker depolarization and AP behavior.

The paper is organized as follows: the model is presented in Section II. Section III shows some simulation results. A discussion and conclusions are presented in Sections VI and $\mathrm{V}$ respectively.

\section{MODEL DESCRIPTION}

The proposed model for the cardiac Purkinje fibres AP is the system (1) of eight first order ordinary differential equations (ODE) (notations are defined in table I):

$$
\left\{\begin{aligned}
\frac{d V}{d t} & =-\frac{I_{K, t}+I_{N a, t}+I_{C a, t}+I_{N a K}+I_{N a C a}}{C_{m}} \\
\frac{d K_{i}}{d t} & =\frac{2 I_{N a K}-I_{K, t}}{F V_{C}} \\
\frac{d N a_{i}}{d t} & =-\frac{I_{N a, t}+3 I_{N a K}+3 I_{N a C a}}{F V_{C}} \\
\frac{d C a_{i}}{d t} & =\frac{2 I_{N a C a}-I_{C a, t}}{2 F V_{C}}+J_{l e a k}+J_{r e l}-J_{u p} \\
& -\sum_{b \in I_{B}} B_{b} \frac{d \theta_{b}}{d t}, \quad I_{B}=\{T n, C a l\}, \\
\frac{d g_{X}}{d t} & =\frac{g_{X \infty}-g_{X}}{\tau_{g X}}, \quad X \in\{N a, K\} \\
\frac{d \theta_{b}}{d t} & =k_{b}^{o n}\left|C a_{i}\right|\left(1-\theta_{b}\right)-k_{T n}^{o f f} \theta_{b}, \quad b \in I_{B}
\end{aligned}\right.
$$

The gate dynamics are defined by

$$
\begin{aligned}
g_{X \infty} & =\frac{1}{2}\left[1+\tanh \left(\frac{V-V_{g_{X}}}{R T / 2 F}\right)\right], \\
\frac{1}{\tau_{g_{X}}} & =\frac{1}{\tau_{X}} \cosh \left(\frac{V-V_{g_{X}}}{R T / 2 F}\right), \quad X \in\{N a, K, d, m\}
\end{aligned}
$$

where $X=d, m$ represent fast $C a, N a$ activation gating, denoted as usual $d_{\infty}=g_{d \infty}, m_{\infty}=g_{m \infty}$. Setting

$$
V_{X}=\frac{R T}{z_{X} F} \log \left|\frac{X_{e}}{X_{i}}\right|, X \in\{C a, N a, K\},
$$

the currents through the membrane are then:

$$
\begin{aligned}
I_{K, t} & =\bar{I}_{K} g_{K} \sinh \left(\frac{V-V_{K}}{2 R T / F}\right)+\bar{I}_{b, K} \\
I_{N a, t} & =\bar{I}_{N a} g_{N a} m_{\infty} \sinh \left(\frac{V-V_{N a}}{2 R T / F}\right) \\
I_{C a, t} & =\left[\bar{I}_{C a}\left(1-g_{K}\right) d_{\infty}+\bar{I}_{b, C a}\right] \sinh \left(\frac{V-V_{C a}}{R T / F}\right) \\
I_{N a K} & =\bar{I}_{N a K} \tanh \left(\frac{V+2 V_{K}-3 V_{N a}-V_{A T P}}{2 R T / F}\right) \\
I_{N a C a} & =\bar{I}_{N a C a} \sinh \left(\frac{V+2 V_{C a}-3 V_{N a}}{2 R T / F}\right)
\end{aligned}
$$

Finally, the CICR mechanism is described using

$$
\begin{aligned}
J_{u p}= & Q_{u p} J_{\text {max }} \frac{\left|\frac{C a_{i}}{K_{m f}}\right|^{H}-\left|\frac{C a_{S R}}{K_{m r}}\right|^{H}}{1+\left|\frac{C a_{i}}{K_{m f}}\right|^{H}+\left|\frac{C a_{S R}}{K_{m r}}\right|^{H}} \\
J_{\text {rel }}= & K_{\text {rel }} d_{\infty}\left(C a_{S R}-C a_{i J C T}\right) \\
J_{\text {leak }}= & K_{\text {leak }}\left(C a_{S R}-C a_{i J C T}\right) \\
C a_{i J C T}= & \frac{B_{J C T} C a_{i}}{\left|C a_{i}\right|+K_{J C T}} \\
C a_{S R}+ & \frac{B_{S R} C a_{S R}}{\left|C a_{S R}\right|+K_{S R}}= \\
& \frac{V_{C}\left(C a_{T}-C a_{i}-\sum_{b \in I_{B}} B_{b} \theta_{b}-C a_{i J C T}\right)}{V_{S R}}
\end{aligned}
$$

TABLE I

NOTATIONS USED IN THE TEXT

\begin{tabular}{|ll|}
\hline \hline Notations & Definitions \\
\hline$V$ & Action potential \\
$V_{\text {ext }}$ & External stimulus voltage \\
$I_{X, t}$ & Total X current through all channels \\
$I_{N a C a}$ & $\mathrm{Na}^{+}-\mathrm{Ca}^{2+}$ exchanger current \\
$I_{\text {NaK }}$ & $\mathrm{Na}^{+}-\mathrm{K}^{+}$pump current \\
$g_{K}$ & Potassium activation gating \\
$g_{N a}$ & Sodium activation gating \\
$d_{\infty}$ & Fast Calcium activation gating \\
$m_{\infty}$ & Fast Sodium activation gating \\
$J_{\text {rel }}$ & Calcium-Induced Calcium Release current \\
$J_{u p}$ & Pump current taking up calcium in the SR \\
$J_{l e a k}$ & Leakage current from SR to the cytoplasm \\
$X_{i}$ & Intracellular concentration of the free ion $X$ \\
$X_{e}$ & External concentration of the ion free $X$ \\
$\mathrm{Ca}$ & Total calcium concentration in the cell \\
$\mathrm{Ca}$ & Free calcium concentration in the SR \\
$\theta_{T n}$ & Fraction of Troponin-C sites bound with Ca \\
$\theta_{\mathrm{Cal}}$ & Fraction of Calmodulin sites bound with Ca \\
$\mathrm{Ca} a_{i J C T}$ & SR Calcium buffered in the junction \\
\hline
\end{tabular}

$$
\begin{gathered}
C a_{T}=\frac{1}{2}\left(\frac{C_{m}}{F V_{C}}\left(V-V_{e x t}\right)-N a_{i}-K_{i}\right) \\
V_{e x t}=-\frac{F V_{C}}{C_{m}}\left(N a_{e}+K_{e}+2 C a_{e}\right)
\end{gathered}
$$

\section{RESULTS}

The system of nonlinear ODE is solved with a second order modified Rosenbrock method with variable steplength. The parameters values are listed in table II. Some simulations of the autorhythmic activity are presented.

Figure 1 shows the simulated Purkinje fibres AP. It presents the characteristic spike, followed by the rapid depolarization, then the potential moves again in a positive direction, thus generating the 'notch' characteristic of many experimental records [2]. The plateau phase after the 'notch' follows a somewhat rounded (concave downwards) time course similar to experimental records [2]. Repolarization terminates followed by the slow spontaneous depolarization (pacemaker potential) until the threshold is reached and another action potential is initiated.

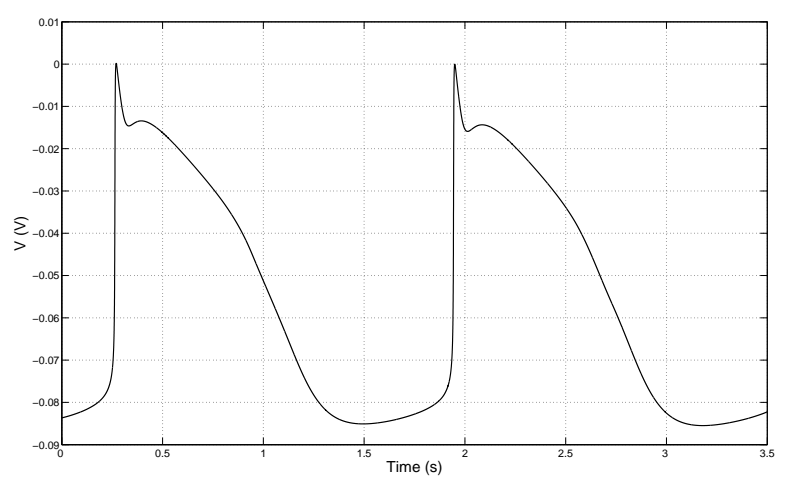

Fig. 1. Simulated Purkinje fibres action potential 

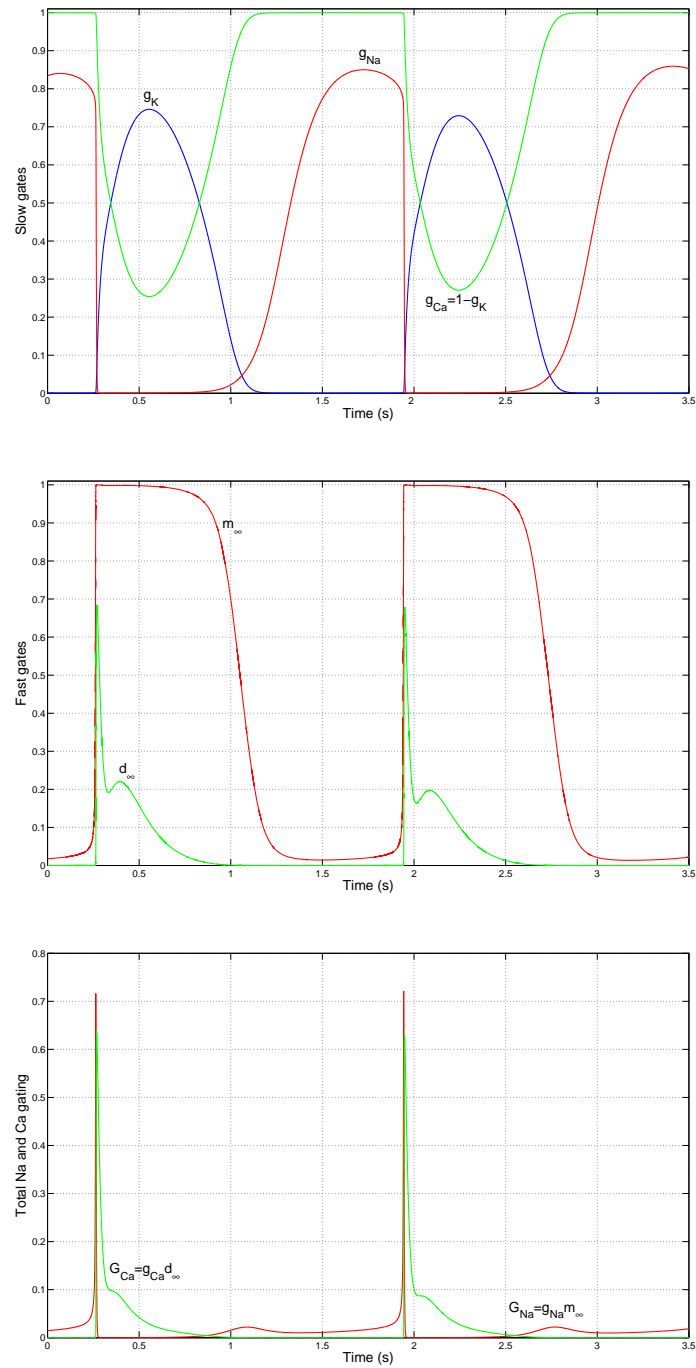

Fig. 2. Top: Simulated slow gating variables. Middle: Simulated fast gating variables. Bottom: Simulated total $\mathrm{Na}$ and $\mathrm{Ca}$ gating variables.

In Fig. 2, the time course of the computed gating variables changes is shown. It can be seen that the slow gates $g_{C a}$ and $g_{N a}$ rise to a high value during the pacemaker depolarization phase $(100 \%$ and $85 \%$ are the fractions of $\mathrm{Ca}$ and $\mathrm{Na}$ channels in the open state respectively). However, the fast gating variables corresponding to the sodium and calcium channels fall (the probability of calcium channels being open is zero and is approximately $2 \%$ for sodium channels). Thus, only the inward sodium current contributes to pacemaker activity. This result is clearly shown in the bottom of the figure 2 and in the top where the slow potassium gating variable falls to zero. Only $\mathrm{Ca}$ and $\mathrm{Na}$ have a quantitative role in the spike action potential phase. By contrast, potassium and calcium play an important role in the plateau and in the repolarization phases (calcium is more important during the plateau than during the repolarization phase).

In Fig. 3, the time courses of the five ionic currents and the intracellular calcium concentration changes are shown.
TABLE II

MODEL PARAMETERS

\begin{tabular}{|c|c|c|}
\hline Parameter & Value & Unit \\
\hline$V_{C}$ & 16.404 & $p L$ \\
\hline$V_{S R}$ & 1.094 & $p L$ \\
\hline$F$ & 96.486 & $\mathrm{Cmmol}^{-1}$ \\
\hline$V_{m}$ & $-56.8610^{-3}$ & $V$ \\
\hline$V_{d}$ & $-510^{-3}$ & $V$ \\
\hline$V_{g_{K}}$ & $-2610^{-3}$ & $V$ \\
\hline$V_{g_{N a}}$ & $-71.5510^{-3}$ & $V$ \\
\hline$\tau_{N a}=\tau_{K}$ & 0.2 & $s$ \\
\hline$V_{A T P}$ & $-45010^{-3}$ & $V$ \\
\hline$C_{m}$ & 85 & $p F$ \\
\hline$R$ & $8.31410^{-3}$ & $\mathrm{Jmmol}^{-1} K^{-1}$ \\
\hline$T$ & 310 & $K$ \\
\hline$z_{N a}=z_{K}$ & 1 & \\
\hline$z_{C a}$ & 2 & \\
\hline $\bar{I}_{N a}$ & 112.7 & $p A$ \\
\hline $\bar{I}_{K}$ & 32.9 & $p A$ \\
\hline $\bar{I}_{b, K}$ & 70 & $p A$ \\
\hline $\bar{I}_{C a}$ & 26.2 & $p A$ \\
\hline $\bar{I}_{b, C a}$ & 0.01645 & $p A$ \\
\hline $\bar{I}_{N a K}$ & 11.46 & $p A$ \\
\hline $\bar{I}_{\mathrm{NaCa}}$ & 1400 & $p A$ \\
\hline$K_{e}$ & 5.4 & $m M$ \\
\hline$N a_{e}$ & 140 & $m M$ \\
\hline$C a_{e}$ & 2 & $m M$ \\
\hline$K_{\text {leak }}$ & $510^{-3}$ & $s^{-1}$ \\
\hline$H$ & 1.787 & \\
\hline$K_{m f}$ & $0.24610^{-3}$ & $m M$ \\
\hline$K_{m r}$ & 1.7 & $m M$ \\
\hline$J_{\max }$ & $28610^{-3}$ & $m M s^{-1}$ \\
\hline$Q_{u p}$ & 2.6 & \\
\hline$K_{\text {rel }}$ & $2510^{3}$ & $s^{-1}$ \\
\hline$k_{T n}^{o n}$ & $32.710^{3}$ & $m M^{-1} s^{-1}$ \\
\hline$k_{T n}^{o f f}$ & 19.6 & $s^{-1}$ \\
\hline$B_{T n}$ & $7010^{-3}$ & $m M$ \\
\hline$k_{\mathrm{Cal}}^{o n}$ & $3410^{3}$ & $m M^{-1} s^{-1}$ \\
\hline$k_{\text {Cal }}^{o f f}$ & 238 & $s^{-1}$ \\
\hline$B_{C a l}$ & $2410^{-3}$ & $m M$ \\
\hline$K_{J C T}$ & $1310^{-3}$ & $m M$ \\
\hline$B_{J C T}$ & $4.610^{-3}$ & $m M$ \\
\hline$K_{S R}$ & $65010^{-5}$ & $m M$ \\
\hline$B_{S R}$ & 0.14 & $m M$ \\
\hline
\end{tabular}

The intracellular $\mathrm{Ca}^{2+}$ transient rises to a peak within a few milliseconds and decays as expected in a biphasic response before the faster phase of repolarization begins. This corresponds well to the experimental results on Purkinje fibres [11]. In principle, two peaks are possible since the $\mathrm{Ca}^{2+}$ is made up of two components: the smaller one due to the calcium current and the larger one due to internal release.

While $I_{N a, t}$ is the main time-dependent gated current that contributes to pacemaker activity, other currents also contribute substantially. The net increase in $I_{N a, t}$ during the pacemaker depolarization in figure 3 is $-15 \mathrm{pA}$. By comparison, $I_{b, C a}$ shows a fall of $-15 \mathrm{pA}$ which produces the fall of the $I_{C a, t}, I_{K, t}$ and $I_{N a C a}$ remains equal to zero during the pacemaker potential, and $I_{\mathrm{NaK}}$ carries about $1.5 \mathrm{pA}$.

\section{Discussion}

In this paper, a model for cardiac Purkinje fibres action potential is proposed. With only eight state variables, it is realistic enough to exhibit many of the characteristics of 

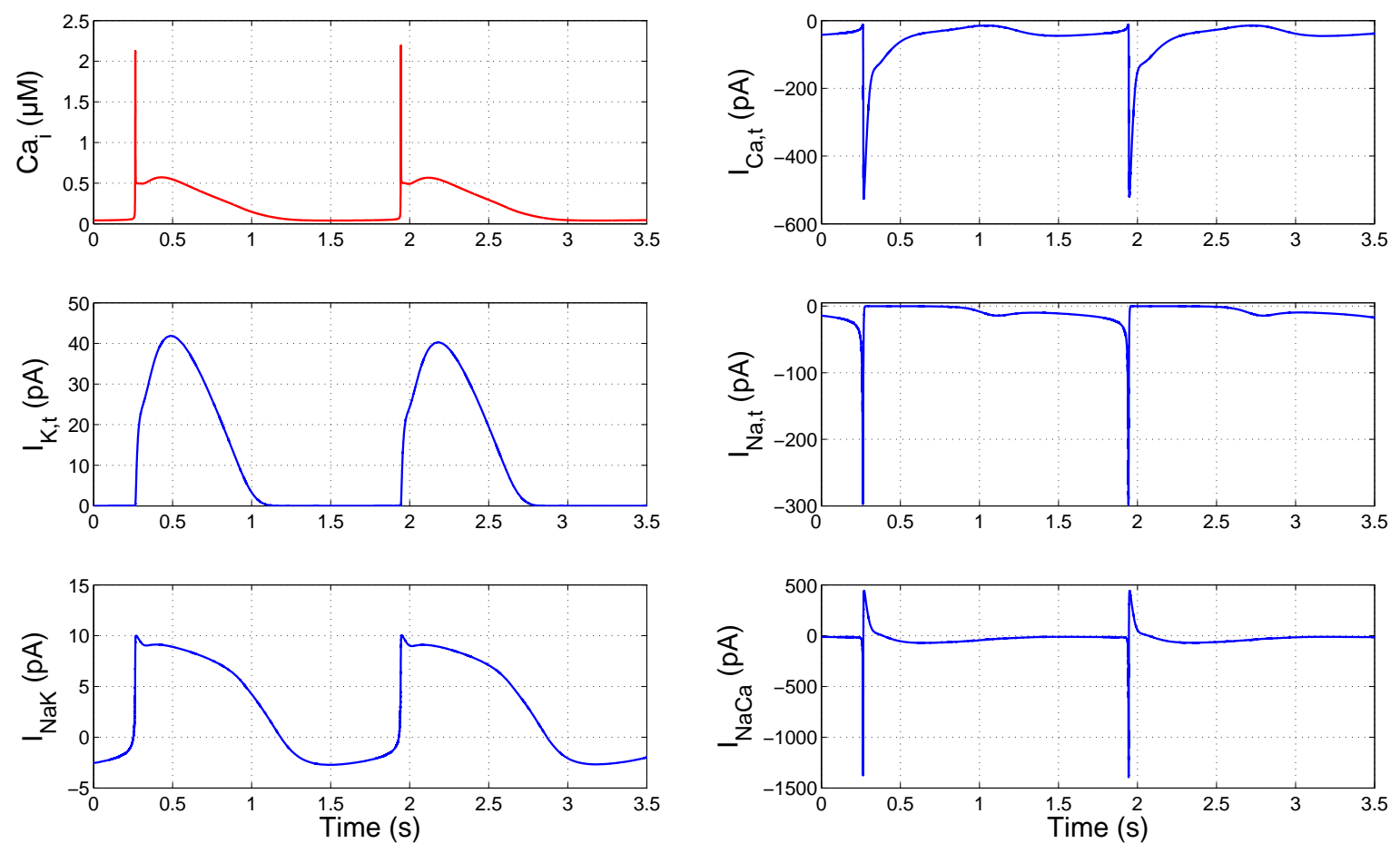

Fig. 3. Simulated intracellular calcium transients and ionic currents

larger pacemaker cell models. Its structure, common with ventricular cardiac cells, involves $\mathrm{Na}^{+}, \mathrm{K}^{+}$and $\mathrm{Ca}^{2+}$ ions, their respective channels, the $\mathrm{Na}^{+} / \mathrm{Ca}^{2+}$ exchanger, and the $\mathrm{Na}^{+} / \mathrm{K}^{+}$pump. It also includes a description of the dynamics of the main calcium buffers in the bulk cytosol and in the SR, taking into account the calcium uptake and release from SR.

The model can be tuned to reproduce Purkinje fibres action potential, the behavior of the main currents and the intracellular calcium dynamics involved during pacemaking.

The mechanisms underlying pacemaker depolarization of Purkinje fibres model were examined by ascertaining the dominant membrane currents during each phase of the action potential. Pacemaker depolarization was found to arise from the net contribution of a number of inward currents, the largest of which was the $\mathrm{Na}^{+}$current. In contrast, the delayed rectifier $K^{+}$does not play a role in pacemaker depolarization, remaining equal to zero throughout most of this phase.

Due to its medium complexity, this model can be used in multi-beat simulations from the cell to the heart scales. It can also be useful in direct computations to gain insights and for its predictive capabilities, and is also a good candidate in inverse problems to estimate state and parameters from measurements.

\section{CONClusion}

We have presented a reduced differential model of the membrane potential, ionic currents and calcium dynamics in a Purkinje fibre cell. It is in good agreement with more complete models. The model represents a good trade-off between descriptive power and complexity. It is a good candidate to be imbedded in heart simulators for applications where both direct and inverse problems are considered.

\section{REFERENCES}

[1] D. Noble, "A modification of the Hodgkin-Huxley equations applicable to Purkinje fibre action and pace-maker potentials," J Physiol, vol. 160, pp. 317-52, 1962.

[2] R. E. McAllister, D. Noble, and R. W. Tsien, "Reconstruction of the electrical activity of cardiac Purkinje fibres," J Physiol, vol. 251, no. 1, pp. 1-59, 1975.

[3] D. DiFrancesco and D. Noble, "A model of cardiac electrical activity incorporating ionic pumps and concentration changes," Philos Trans R Soc Lond B Biol Sci, vol. 307, no. 1133, pp. 353-98, 1985.

[4] CardioSense3D, "An INRIA Large Initiative Action," //wwwsop.inria.fr/CardioSense3D, 2006.

[5] K. Djabella and M. Sorine, "A differential model of excitationcontraction coupling in a cardiac cell for multicycle simulations," in Proceedings of the 3rd European Medical and Biological Engineering Conference, Prague, Czech Republic, November 20-25 2005.

[6] L. P. Endresen, K. Hall, J. S. Hoye, and J. Myrheim, "A theory for the membrane potential of living cells," Eur Biophys $J$, vol. 29, pp. 90-103, 2000.

[7] T. J. Hund, J. P. Kucera, N. F. Otani, and Y. Rudy, "Ionic charge conservation and long-term steady state in the Luo-Rudy dynamic cell model," Biophys Journal, vol. 81, pp. 3324-3331, 2001.

[8] K. H. W. J. T. Tusscher, D. Noble, P. J. Noble, and A. V. Panfilov, "A model for human ventricular tissue," Am J Physiol Heart Circ Physiol, vol. 286, pp. 1573-1589, 2004.

[9] T. R. Shannon, F. Wang, J. Puglisi, C. Weber, and D. M. Bers, "A mathematical treatment of integrated Ca dynamics within the ventriclar myocite," Biophys Journal, vol. 87, pp. 3351-3371, 2004.

[10] D. M. Bers, "Calcium and cardiac rhythms: Physiological and pathophysiological," Circ Res, vol. 90, pp. 14-17, 2002.

[11] W. G. Wier and G. Isenberg, "Intracellular [Ca] transients in voltageclamped cardiac Purkinje fibres," Pflugers Arch. Eur. J. Physiol, vol. 392, pp. 284-290, 1982. 